\title{
Architectural RNAs for Membraneless Nuclear Body Formation
}

\author{
Tomohiro Yamazaki, ${ }^{1}$ Shinichi Nakagawa, ${ }^{2}$ and Tetsuro Hirose ${ }^{1}$ \\ ${ }^{1}$ Institute for Genetic Medicine, Hokkaido University, Sapporo, 060-0815 Japan \\ ${ }^{2}$ Faculty of Pharmaceutical Sciences, Hokkaido University, Sapporo, 060-0812 Japan \\ Correspondence: hirose@igm.hokudai.ac.jp
}

\begin{abstract}
Long noncoding RNAs (lncRNAs) are fundamental regulators of various cellular processes. A subset of lncRNAs, termed architectural RNAs (arcRNAs), function in the formation and maintenance of phase-separated membraneless organelles in multiple eukaryotic species. These membraneless organelles represent an important type of compartmentalization in the crowded cellular environment and have several distinct features. The NEAT1_2 lncRNA is a well-characterized arcRNA that functions as an essential scaffold of paraspeckle nuclear bodies. Here, we describe the biogenesis of paraspeckles on arcRNAs through phase separation, focusing on the specific functions of multiple NEAT1_2 RNA domains and their partner RNAbinding proteins. Finally, we present an updated model of paraspeckle formation and discuss future perspectives of research into arcRNA-instructed architectures of phase-separated nuclear bodies.
\end{abstract}

\section{ARCHITECTURAL RNAS ARE ESSENTIAL SCAFFOLDS OF PHASE-SEPARATED MEMBRANELESS BODIES}

Recently, membraneless organelles and biomolecular condensates, which form through phase separation, have attracted increasing interest (Banani et al. 2017; Alberti et al. 2019). These subcellular compartments play key roles in a number of processes, including the sequestration of biomolecules and the formation of organization hubs to coordinate biochemical reactions and macromolecular assembly (Shin and Brangwynne 2017). A subset of phaseseparated cellular bodies are constructed by specific long noncoding RNAs (lncRNAs) (Yamazaki and Hirose 2015; Chujo et al. 2016; Chujo and Hirose 2017), which play diverse regulatory roles in various biological processes, including gene expression (Guttman and Rinn 2012; Geisler and Coller 2013; Quinn and Chang 2016; Schmitt and Chang 2017). Multiple lncRNAs can act as essential architectural scaffolds of nuclear bodies in a variety of eukaryotes, including humans, mice, Drosophila, and yeast; hence, we termed them architectural RNAs (arcRNAs) (Yamazaki and Hirose 2015; Chujo et al. 2016; Chujo and Hirose 2017).

A number of arcRNAs are involved in the formation of various nuclear bodies. For example, NEAT1_2, the long isoform of the nuclear paraspeckle assembly transcript 1 (NEAT1) lncRNA, is an essential scaffold of paraspeckle nuclear bodies (Chen and Carmichael 2009; Clemson et al. 2009; Sasaki et al. 2009; Sunwoo et al. 2009); highly repetitive satellite III (HSATIII) lncRNAs, which are induced by conditions such as thermal stress, promote the formation of nuclear stress bodies (nSBs) in primates (Jolly et al. 1999; Aly et al. 2019; Ninomiya et al. 2019);
HSATII RNAs are core components of cancer-associated satellite transcript bodies (Hall et al. 2017); intergenic spacer IncRNAs induce the formation of static but reversible amyloid-like solid structures termed amyloid bodies (Audas et al. 2016); histone pre-mRNAs are essential for histone locus body formation (Shevtsov and Dundr 2011); tumor-associated NBL2 transcript (TNBL) aggregates are induced by TNBL RNAs (Dumbović et al. 2018); the perinucleolar compartment is induced by the PNCTR RNA (Yap et al. 2018); Hsrw RNAs are essential cores of $\omega$-speckles in Drosophila (Mallik and Lakhotia 2009); and the Mei2 dot is a nuclear body induced by meiRNA in fission yeast (Yamashita et al. 1998). The Sam68 nuclear body and DBC1 bodies are also RNA-dependent bodies, but their arcRNAs have not yet been identified (Mannen et al. 2016). In addition, disease-associated repeat RNAs can also be regarded as a type of arcRNA (Zhang and Ashizawa 2017).

The existence of arcRNAs in a large number of organisms suggests that they are suitable for nucleating subnuclear bodies through phase separation. RNA-binding proteins (RBPs) are enriched with prion-like domains (PLDs), low-complexity domains (LCDs), and intrinsically disordered regions (IDRs), which form weak multivalent molecular interactions that promote biological phase separation (Hennig et al. 2015; Yamazaki and Hirose 2015). Thus, arcRNAs can induce phase separation by increasing the local concentration of these RBPs. More than 1000 human RBPs have been reported to date; these proteins possess a wide variety of biological functions, such as transcriptional regulation and epigenetic chromatin regulation (Baltz et al. 2012; Castello et al. 2012, 2016). RNAs can assemble a specific set of RBPs to combine and integrate their functions, even when the RBPs

(C) 2019 Yamazaki et al. This article is distributed under the terms of the Creative Commons Attribution-NonCommercial License, which permits reuse and redistribution, except for commercial purposes, provided that the original author and source are credited. 
would not directly interact (Chujo et al. 2016; Engreitz et al. 2016). This property allows arcRNA-induced bodies to perform multiple tasks in the cell, including acting as molecular sponges to sequestrate proteins and/or RNAs, forming reaction crucible to enhance biochemical reactions, and organizational hubs of chromatin (Chujo et al. 2016; Chujo and Hirose 2017; Hirose et al. 2019). In addition, noncoding RNAs can act as functional molecules themselves, with roles that depend on their specific sequences and do not require protein coding capacity. Architectural noncoding RNAs are able to alter their specific sequences to adapt to circumstantial changes. Furthermore, multiple copies of lncRNAs are synthesized from their gene loci; this process is important for phase separation as it promotes multivalent interactions between high local concentrations of associated IDR proteins. Therefore, lncRNAs act as markers of specific genomic loci. This property of lncRNAs is beneficial to the amplification of signals from specific genomic loci, such as superenhancers, and phase separation would enhance this effect (Engreitz et al. 2016). Transcription of arcRNAs is essential for the formation of phase-separated nuclear bodies, and inhibition of their transcription leads to rapid disinte- gration of the bodies. As most arcRNAs are stress-inducible or specifically expressed in certain developmental and pathological conditions, phase-separated nuclear bodies are transiently formed upon the transcription of arcRNAs. Therefore, it is conceivable that arcRNAs spatiotemporally control cellular signals and act as integral hubs for gene regulation.

In the sections below, we focus on NEAT1_2 as a model arcRNA. We describe how NEAT1_2 arcRNAs construct paraspeckle nuclear bodies and determine their biophysical properties, with a special focus on the functional RNA domains of NEAT1 and their partner proteins.

\section{THE CHARACTERISTICS OF PHASE- SEPARATED PARASPECKLE NUCLEAR BODIES CONSTRUCTED BY THE NEAT1_2 arcRNA}

Paraspeckles were originally identified as distinct nuclear bodies called interchromatin granule-associated zones (IGAZs) (Visa et al. 1993), which were located adjacent to interchromatin granule clusters (also known as nuclear speckles) (Fig. 1A). In 2002, paraspeckles were identified
A

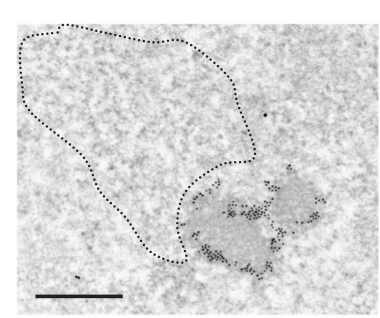

B

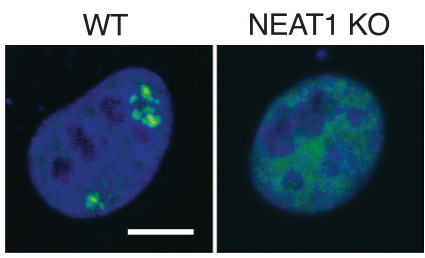

C

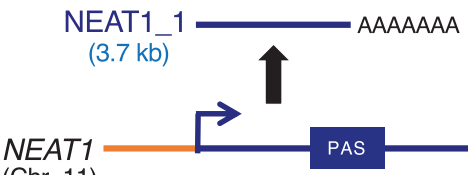

(Chr. 11)

NEAT1_2 $(22.7 \mathrm{~kb})$

D

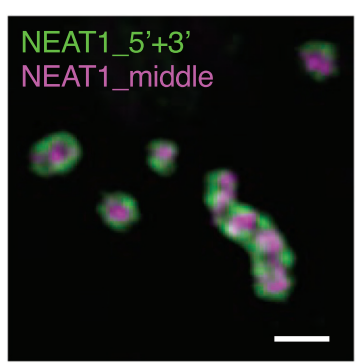

E

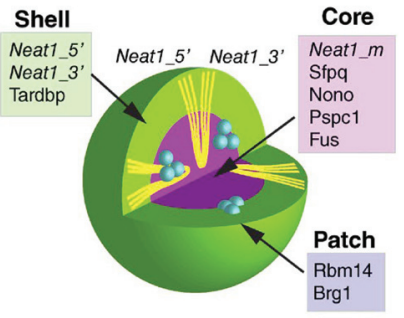

Figure 1. The NEAT1 2 architectural RNA (arcRNA) is essential for maintaining paraspeckle integrity. $(A)$ An electron microgram of a paraspeckle (electron dense structures were detected using gold particles [probes against NEAT1 $5^{\prime}$ regions]) and nuclear speckles (black dashed circle) in human HAP1 cells treated with MG132 $(5 \mu \mathrm{M})$ for $17 \mathrm{~h}$. Scale bar, $500 \mathrm{~nm}$. The image was kindly provided by Dr. Gerard Pierron (Institut Gustave Roussy, CNRS). (B) Visualization of paraspeckles in wild-type (WT) and NEAT1 knockout human cells by NONO immunofluorescence (green foci). The diffuse green signals seen in the knockout cells indicate disruption of proper paraspeckle formation. DNA was stained with DAPI (blue). Scale bar, $5 \mu \mathrm{m}$. (C) Schematic illustration of the NEAT1_1 and NEAT1_2 isoforms of the NEAT1 gene. $(D)$ A super-resolution microscopy image (structured illumination microscopy) of paraspeckles stained with a NEAT1 FISH probe (green, the 5' and 3' regions; magenta, the middle region). Scale bar, $500 \mathrm{~nm}$. (E) A schematic illustration of NEAT1_2 configuration within a paraspeckle and the core-shell-patch localizations of paraspeckle-localizing proteins (PSPs). 
as nuclear bodies enriched in PSPC1, a member of the Drosophila behavior human splicing (DBHS) family of proteins that includes SFPQ and NONO (Fig. 1B, left; Fox et al. 2002). Subsequently, paraspeckles were shown to be identical to IGAZs and were found to be RNasesensitive structures, suggesting a requirement of RNAs for their maintenance (Fox et al. 2005). In 2009, four groups independently reported that the NEAT1 lncRNA, also called Menc/ $\beta$, is an essential architectural scaffold of paraspeckles, as shown by a lack of discrete paraspeckles in NEAT1 knockdown cells (Fig. 1B, right; Chen and Carmichael 2009; Clemson et al. 2009; Sasaki et al. 2009; Sunwoo et al. 2009). NEAT1 has two isoforms: the longer NEAT1_2/Men $\beta$ isoform (22.7 $\mathrm{kb}$ in human) is essential for paraspeckle formation, whereas the short NEAT1_1/ Menc isoform (3.7 kb in human) is dispensable (Fig. $\overline{1 C}$; Naganuma et al. 2012; Yamazaki et al. 2018a,b).

Several subsequent studies identified more than 60 paraspeckle-localizing proteins (PSPs) in humans (Naganuma et al. 2012; Fong et al. 2013; Kawaguchi et al. 2015; Yamazaki and Hirose 2015; Mannen et al. 2016). Most PSPs are RBPs that contain PLDs, LCDs, or IDRs that show significant overlaps with a number of genes associated with amyotrophic lateral sclerosis (Yamazaki and Hirose 2015). Electron microscopy studies revealed that paraspeckles are roundish or oblong structures with constant short axes ( $\sim 360 \mathrm{~nm}$ in human HeLa cells) (Fig. $1 \mathrm{~A})$ and become elongated when NEAT1_2 is up-regulated (Souquere et al. 2010; Hirose et al. 2014b). Notably, the NEAT1_2 lncRNA is spatially organized within paraspeckles; the 5' and 3 ' regions of NEAT1_2 are located in the shells of paraspeckles, whereas the middle region is located in the core region, suggesting that NEAT1_2 forms a looped structure within paraspeckles (Fig. 1 $\bar{A}$, D; Souquere et al. 2010; West et al. 2016). In addition, PSPs are also spatially organized within paraspeckles, with distinct localizations at the core, shell, and patch regions (Fig. 1E; Kawaguchi et al. 2015; West et al. 2016).
Multiple lines of evidence support the concept that paraspeckles are phase-separated nuclear bodies. First, fluorescence recovery after photobleaching (FRAP) experiments revealed that PSPs are dynamically exchanged between paraspeckles and the surrounding nucleoplasm (Mao et al. 2011; Audas et al. 2016; Wang et al. 2018). Second, paraspeckles are sensitive to 1,6-hexanediol, which mainly disrupts hydrophobic interactions and contributes to the formation of phase-separated subcellular bodies (Yamazaki et al. 2018b). Third, the abilities of the PSPs FUS and RBM14, which are essential for paraspeckle formation, to form hydrogels in vitro are essential for paraspeckle assembly (Hennig et al. 2015; Fox et al. 2018). Fourth, paraspeckles undergo a fusion and fission process termed "kiss-and-run fusion" (Mao et al. 2011; Yang et al. 2019). A recent review article reported that paraspeckles are induced by polymer-polymer phase separation rather than the typical liquid-liquid phase separation (Peng and Weber 2019). Taken together, these findings suggest that paraspeckles are massive phase-separated subnuclear structures with a distinct shape and ordered interior configuration.

\section{BIOGENESIS OF THE NEAT1_2 arcRNA AND PARASPECKLES}

Synthesis of the NEAT1 isoforms is regulated by alternative 3 '-end processing governed by HNRNPK and TDP-43 (Naganuma et al. 2012; Modic et al. 2019). The 3 ' end of NEAT1_2 contains an unusual triple helix structure $\left(3^{\prime} \mathrm{TH}\right)$ that is essential for stabilization of the arcRNA (Fig. 1C; Brown et al. 2012; Wilusz et al. 2012; Yamazaki et al. 2018b). Seven PSPs and the SWI/SNF complex are essential for paraspeckle formation (Fig. 2; Naganuma et al. 2012; Kawaguchi et al. 2015; West et al. 2016). Specifically, SFPQ, NONO, and RBM14 are essential for NEAT1_2 stability (Naganuma et al. 2012), and HNRNPK controls isoform switching to the NEAT1_2 isoform by inhibiting polyadenylation of the NEAT1_1

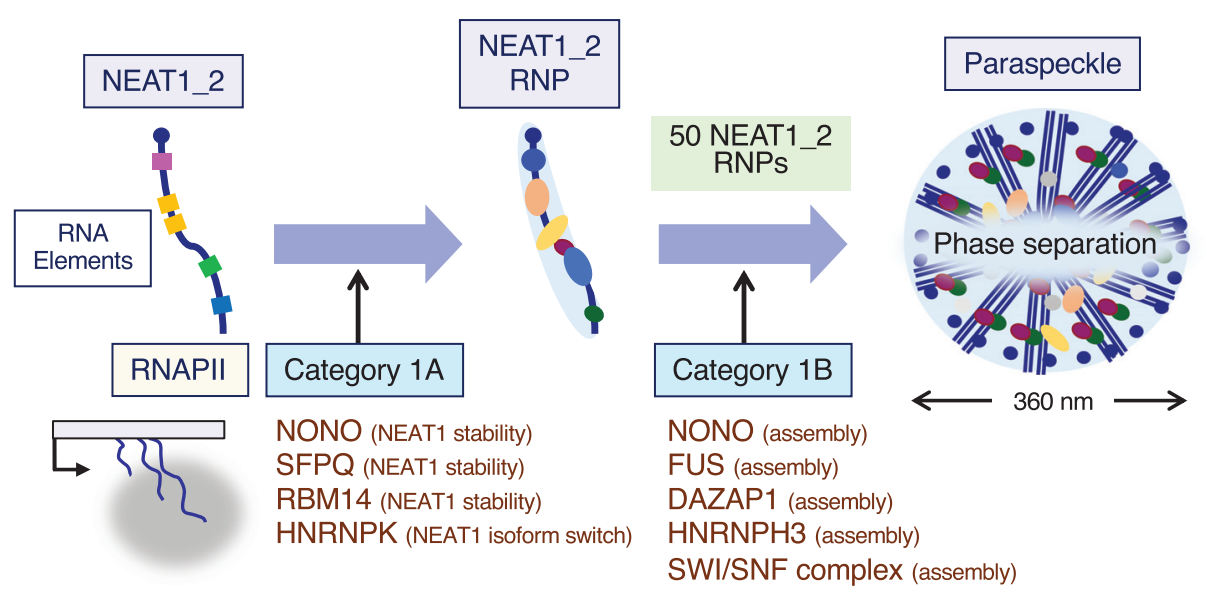

Figure 2. Two distinct processes of paraspeckle formation. NEAT1_2 ribonucleoproteins (RNPs) are transcribed by RNA polymerase II (RNAPII) and then approximately 50 NEAT1_2 RNPs assemble into paraspeckles through phase separation. PSPs that are essential for paraspeckle integrity can be divided into two categories: category 1A includes PSPs required for NEAT1_2 expression and category 1B includes PSPs required for paraspeckle assembly. Putative RNA elements are shown on the NEAT1_2 structure (left). 
isoform (Fig. 2; Naganuma et al. 2012). NONO, FUS, DAZAP1, HNRNPH3, and the SWI/SNF complex are essential for paraspeckle assembly (Naganuma et al. 2012; Kawaguchi et al. 2015; Yamazaki et al. 2018b), and RNA polymerase II transcription is also essential for paraspeckle formation (Fig. 2). Upon transcriptional inhibition, paraspeckles are rapidly disassembled alongside NEAT1_2 degradation and relocation of PSPs to nucleolar cap structures (Fox et al. 2005; Chujo et al. 2017). Paraspeckles are formed in close proximity to the NEAT1 gene loci, likely because NEAT1_2 lncRNAs are highly concentrated at these regions during transcription, and thus the environment favors phase separation by facilitating molecular interactions among RNAs and proteins. This evidence suggests cotranscriptional formation of paraspeckles via phase separation (Mao et al. 2011; Yamazaki et al. 2018b). During this process, approximately 50 NEAT1_2 lncRNAs are incorporated into a single spherical paraspeckle (Fig. 2; Chujo et al. 2017). The paraspeckle is a highly dynamic structure; FRAP studies revealed that $60 \%-70 \%$ of PSPs within paraspeckles are recovered within several minutes (Mao et al. 2011; Audas et al. 2016; Wang et al. 2018). In addition, a recent study showed that elongated, oblong paraspeckles show slower mobility than spherical paraspeckles (Wang et al. 2018). These data indicate the existence of slow or immobile fractions of paraspeckles and that paraspeckle dynamics are related to their shape.

\section{FUNCTIONAL RNA DOMAINS OF THE NEAT1_2 arcRNA}

Although the biological importance of various lncRNAs has been shown, the specific sequences and structural elements required for their biogenesis and functions remain poorly understood (Hirose et al. 2014a). Nonetheless, some lncRNAs have been studied intensively. A previous study examining the XIST lncRNA revealed the existence of functional modular repeat domains involved in X-chromosome inactivation (Wutz et al. 2002). Subsequent studies identified the subdomains of the XIST lncRNA and their partner proteins required for specific functions (Chu et al. 2015; Monfort and Wutz 2017; Colognori et al. 2019). We recently identified multiple modular domains of NEAT1 involved in its biogenesis and the formation of paraspeckles (Yamazaki et al. 2018b). Similar to that of many other lncRNAs, the cross-species homology of mammalian NEAT1_2 sequences is markedly lower than those of protein-coding mRNAs. In general, mammalian NEAT1_2 sequences only display homology at the $5^{\prime}$ region $(\sim 1.6 \mathrm{~kb})$, short stretches in the internal regions, and the 3'TH (Fig. 3A, B). Nevertheless, mouse Neat1_2 can form paraspeckles in human cells, suggesting that the functions of NEAT1_2, at least in paraspeckle formation, are conserved between mouse and human (Fig. 3C; Naganuma et al. 2012).
A

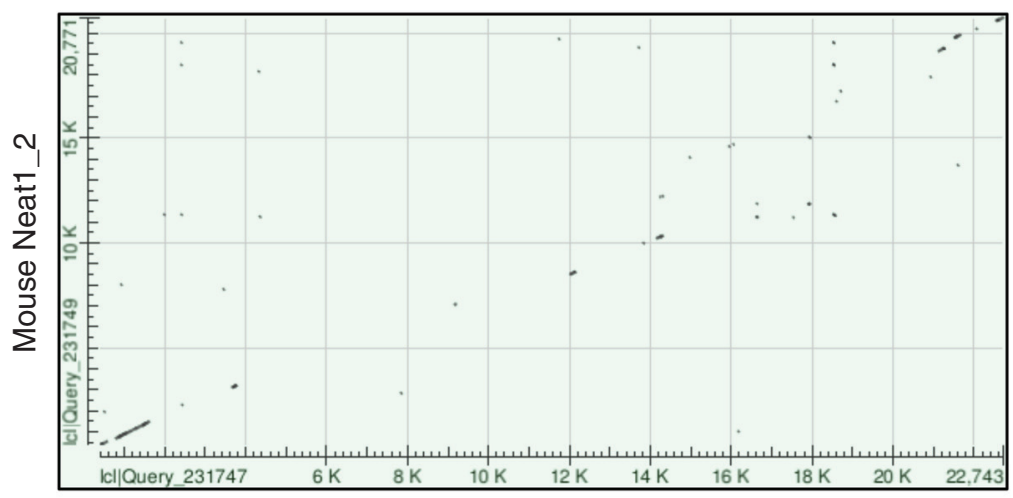

Human NEAT1_2
C

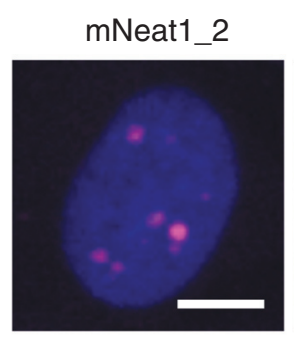

HeLa

B

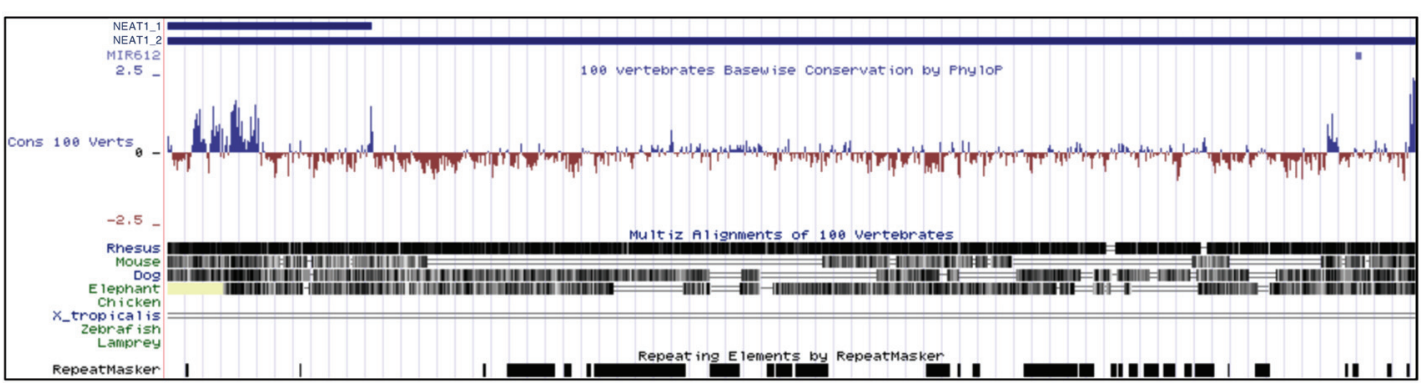

Figure 3. Conservation of the sequences and functions of NEAT1_2 between human and mouse. $(A)$ A dot blot showing the homologous regions of the human and mouse NEAT1 2 sequences. The BLAST search parameter was "somewhat similar." $(B)$ A screenshot of the UCSC genome browser record for the human NEAT1 gene locus with modifications. Sequence conservation (PhastCons) and positions of repetitive elements identified by a RepeatMasker algorithm are shown. $(C)$ Transient expression of mouse Neat 12 (mNeat1 2) induces paraspeckles in human HeLa cells (magenta signals). Scale bar, $5 \mu \mathrm{m}$. 
To dissect the roles of specific sequences within the human NEAT1_2 lncRNA, we performed extensive deletion analyses using CRISPR-Cas9 and the human haploid HAP1 cell line (Yamazaki et al. 2018b). These analyses revealed that multiple functional RNA domains of NEAT1_2 dictate its own biogenesis and the coupled formation of paraspeckles. The 5' end (first $1 \mathrm{~kb}$ ) and 3'TH of NEAT1_2 (termed the A domains) are necessary for stability of the lncRNA (Yamazaki et al. 2018b). The regions upstream $(2.1-2.8 \mathrm{~kb})$ and downstream $(4-5 \mathrm{~kb})$ of the polyadenylation site of NEAT1_1 (termed the B domains) promote the expression of NEAT1_2 by repressing NEAT1_1 polyadenylation, indicating a role in isoform switching from NEAT1_1 to NEAT1_2 (Yamazaki et al. 2018b). The middle domain of NEAT̄1_2 (termed the C domain) is necessary and sufficient for paraspeckle assembly. Notably, our CRISPR dissection analysis also revealed that the $\mathrm{C}$ domain contains several subdomains ( $\mathrm{C} 1$ : 9.8$12 \mathrm{~kb}$; $\mathrm{C} 2: 12-13 \mathrm{~kb}$; C3: $15.4-16.6 \mathrm{~kb})$ that have redundant functions in paraspeckle assembly (Yamazaki et al. 2018 b). Such redundancy is also found among the C domain and the unidentified outside domain, suggesting that the presence of multiple redundant domains confers a robust competency of NEAT1_2 to build paraspeckles (Fig. 4).

In our recent study, artificial tethering of NONO, SFPQ, or FUS, but not RBM14, rescued paraspeckle assembly in cells expressing a NEAT1 mutant lacking the $\mathrm{C} 1$ and $\mathrm{C} 2$ subdomains (Yamazaki et al. 2018b). Moreover, the rescue activity of NONO required dimerization with DBHS family proteins, including SFPQ, PSPC1, and NONO itself, which is also required for the oligomerization of these proteins along the RNA molecule. CLIP-seq data showed that the $\mathrm{C}$ domain of NEAT1_2 contains multiple binding sites for NONO/SFPQ, which are likely to be essential for paraspeckle formation (Naganuma et al. 2012; Passon et al. 2012; Lee et al. 2015; Huang et al. 2018; Yamazaki et al. $2018 b$ ). The in vitro-transcribed RNA derived from the C2 domain preferentially binds to NONO/SFPQ and induces the formation of higher-order structures that are sensitive to 1,6-hexanediol and depend on NONO/SFPQ (Yamazaki et al. 2018b). In addition, the NONO/SFPQ oligomer seems to recruit additional essential PSPs such as FUS and RBM14, which in turn likely induce phase separation (Hennig et al. 2015; Yamazaki et al. 2018b). We recently found that the NEAT1_2 arcRNA is poorly extracted by conventional RNA purification methods using acid guanidinium thiocyanate-phenol-chloroform reagents such as TRIzol (Thermo Fisher Scientific). This unusual feature, which we termed "semiextractability," depends on NONO, FUS, or the NEAT1_2 C domain, suggesting that the tenacious interactions $\bar{b}$ etween these proteins and the $\mathrm{C}$ domain, which can probably tolerate guanidium thiocyanate and phenol-containing harsh conditions, contribute to arcRNA-induced cellular body formation (Chujo et al. 2017; T Yamazaki, unpubl. observ.). SFPQ forms higher-order structures by cooperative spreading and coating the nucleic acids, and a similar spreading mechanism is also proposed to play a role in splicing regulation by HNRNPA1 (Zhu et al. 2001; Lee et al. 2015). Hence, this spreading mechanism might play an important role in the initial process of paraspeckle formation.

In addition to the $\mathrm{A}, \mathrm{B}$, and $\mathrm{C}$ domains, NEAT1_2 contains UG repeat stretches that are evolutionally conserved among multiple species. These repeat sequences are thought to be the major binding sites of TDP-43 (Tollervey et al. 2011). When we deleted the UG repeats from NEAT1_2 using CRISPR-Cas9, recruitment of TDP-43 to the paraspeckles was reduced dramatically (Modic et al. 2019). Furthermore, knock-in of a long UG repeat stretches into the UG-deleted NEAT1_2 mutant recovered the recruitment of TDP-43, suggesting that the UG repeat stretches are necessary and sufficient for TDP-43 recruitment to paraspeckles (Fig. 4; Modic et al. 2019).

As described above, NEAT1_2 is looped and spatially organized within paraspeckles (Fig. 4). Our super-resolution microscopy analyses revealed that deletion of the $3^{\prime}$ region of NEAT1_2 $(\Delta 16.6-22.6-\mathrm{kb}$ mutant $)$ alters its configuration (Yamazaki et al. 2018b). In wild-type cells, the $3^{\prime}$ end of NEAT1_2 is located in the shell of the paraspeckle, but the $3^{\prime}$ end of the $\Delta 16.6-22.6-\mathrm{kb}$ mutant localizes to the paraspeckle core. This finding suggests that the configuration of NEAT1_2 is determined by one or more of its domains. By extension, we speculate that other NEAT1_2 domains will also contribute to the organization of the lncRNA within paraspeckles.

NEAT1_1, which can be regarded as one of the functional domains of the NEAT1 gene, forms "microspeckles," suggesting a role outside of paraspeckles (Li et al. 2017a). A recent study showed that NEAT1_1-specific knockout mice do not show an aberrant phenotype, although a function of NEAT1_1 under specific conditions cannot be excluded (Adriaens et al. 2019; Isobe et al. 2019). Further investigations may reveal specialized roles of NEAT1_1 under certain cellular conditions.

Several recent studies have suggested the presence of additional functional NEAT1 domains. Genome-wide

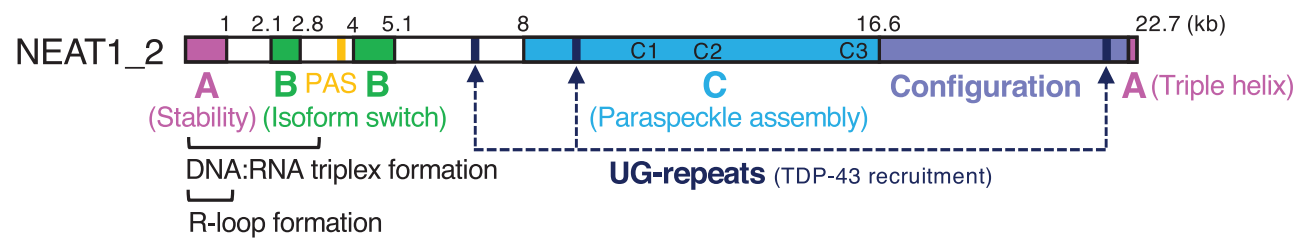

Figure 4. The functional modular domain structure of the human NEAT1_2 arcRNA. The functional domains of NEAT1_2 and their positions (kb, kilobases from the 5' end of NEAT1_2) are shown. (PAS) Polyadenylation signal. 
mapping of R-loop-forming regions revealed that the 5'terminal region of NEAT1 ( 1400 nt) forms a long R-loop (DNA-RNA hybrid), although its biological importance remains unknown (Fig. 4; Dumelie and Jaffrey 2017). Another recent study showed that the 5'-terminal regions of NEAT1, including the GA- and CU-rich regions, form DNA:RNA triplex structures with many human genomic regions (Fig. 4; Senturk Cetin et al. 2019). Consistent with these two reports, the 5 -terminal sequence of NEAT1 is evolutionarily conserved (Fig. 3A), suggesting a sequence-based mechanism for the biological functions of NEAT1, including target gene recognition. In addition, the 3 '-terminal region of human NEAT1_2 harbors a pseudomicroRNA (miR-612) that is not processed into mature microRNA (miRNA) but attracts microprocessors to process pri-miRNAs to pre-miRNAs in the nucleus (Jiang et al. 2017). Overall, as described in this section, the multiple modular domains of NEAT1 determine its biogenesis and the composition, biophysical properties, and functions of paraspeckles.

\section{CURRENT MODEL OF PARASPECKLE FORMATION}

Based on the studies described above, we propose a model of paraspeckle formation (Fig. 5). In this model, NEAT1_2 lncRNAs are transcribed by RNA polymerase II and PSPs associate with nascent NEAT1_2 transcripts at the NEAT1 gene loci (Fig. 5, step (1); Mao et al. 2011). PSPs such as NONO and SFPQ, which are essential for paraspeckle assembly, are loaded onto the multiple functionally redundant high-affinity binding sites of NEAT1_2 and then spread and coat NEAT1_2 domains to form base ribonucleoprotein (RNP) complexes through the NOPS and coiled coil domains of NONO and SFPQ (Fig. 5, steps (2) and (3); Lee et al. 2015; Yamazaki et al. 2018b). Subsequently, the NEAT1_2 RNPs further assemble into paraspeckles (50 NEAT1_2 RNPs per paraspeckle sphere), likely through phase separation. Concurrently, FUS and RBM14 are recruited to the RNPs, likely by NONO and SFPQ, and promote paraspeckle assembly via multivalent interactions between their PLDs (Fig. 5, steps (4) and (5); Hennig et al. 2015; West et al. 2016; Chujo et al. 2017; Yamazaki et al. 2018b). During this process, NEAT1_2 RNPs are bundled and folded (Fig. 5, step (5); West et al. 2016).

It is possible that NEAT1_2 IncRNAs interact with each other via direct intermolecular RNA-RNA interactions to contribute to the phase separation process (Fig. 5, step (2); Van Treeck and Parker 2018). This possibility is supported by the fact that weak NEAT1_2 focal signals remain detectable when PSPs are almost completely obliterated by 1,6-hexanediol treatment (Yamazaki et al. 2018b). Several studies have highlighted the importance of RNA-RNA interactions in the formation of cellular bodies, including stress granules, and disease-associated RNA foci caused by aberrant repeat expansions. It is proposed that the sum of the RNA-RNA, RNA-protein, and protein-protein interactions determines whether arcRNA- protein complexes undergo phase separation that eventually leads to cellular body formation (Van Treeck and Parker 2018).

\section{FUTURE PERSPECTIVES}

Despite recent advances in our understanding of how the NEAT1_2 domains and their partner proteins dictate paraspeckle formation and functions, many questions still remain. First, although NONO and SFPQ have been identified as partner proteins of the NEAT1_2 C domain, it is important to identify the partner RBPs and specific RNA motifs (e.g., sequences and/or secondary structures) required for proper functioning of the other NEAT1_2 functional domains, especially those involved in inducing phase separation. Second, as NEAT1_2 is involved in various physiological and pathological conditions, it is important to investigate how the molecular functions driven by specific RNA domains/motifs link to physiological functions.

We identified NONO and SFPQ as partner proteins of the NEAT1_2 C domain based on prior knowledge of the protein composition of paraspeckles and the results of experiments using immunofluorescence analyses of PSPs on NEAT1 mutants, MS2 tethering, CLIP-seq, and in vitro RNA pulldown (Yamazaki et al. 2018b). These approaches should also enable the identification of partner proteins of the other functional NEAT1_2 domains. To narrow down the functional domains of NEAT1_2 to precise RNA motifs, a large CLIP-seq data set, including eCLIP data, would be a great resource to differentiate between direct and indirect binding proteins and determine their binding sites (Van Nostrand et al. 2016). Analyses of the secondary structure of NEAT1_2 would also be important (Lu et al. 2016; Lin et al. 2018). For example, it would be useful to identify the specific RNA motifs in the NEAT1_2 C domain required for paraspeckle assembly via phase separation. It would also be interesting to understand how specific NEAT1_2 motifs are conserved between species. Furthermore, it would be helpful to understand how the specific domains and motifs within NEAT1_2 can initiate cellular body formation and determine their properties and functions.

Various molecular and physiological or pathological functions of NEAT1_2 have been reported. One of the molecular functions of paraspeckles is to regulate gene expression by acting as molecular sponges that incorporate specific RBPs and RNAs such as IRAlu-containing RNAs, CTN RNA, AG-rich RNAs, and miRNAs (Chen and Carmichael 2009; Hirose et al. 2014b; Imamura et al. 2014; West et al. 2016; Jiang et al. 2017). In addition, the global association of NEAT1/paraspeckles with chromatin has been shown by multiple methods, including CHART-seq, ChIA-PET, GRID-seq, and RADICL-seq (West et al. 2014; Cai et al. 2016; Li et al. 2017b; Bonetti et al. 2019). Under physiological conditions, NEAT1_2 is required for the establishment of pregnancy, mammary gland development, mitochondrial functions, and efficient regulation of gene expression for pluripotency and 
(1)

(2)
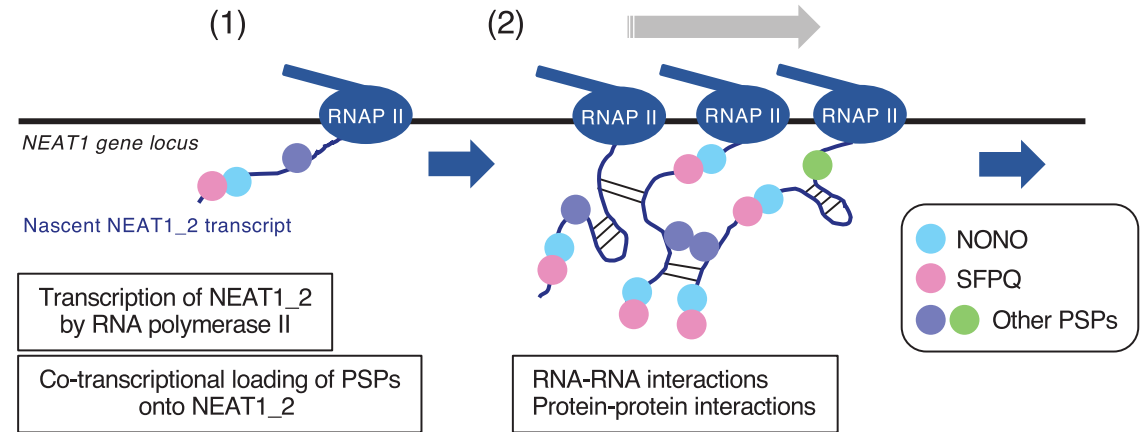

(3)
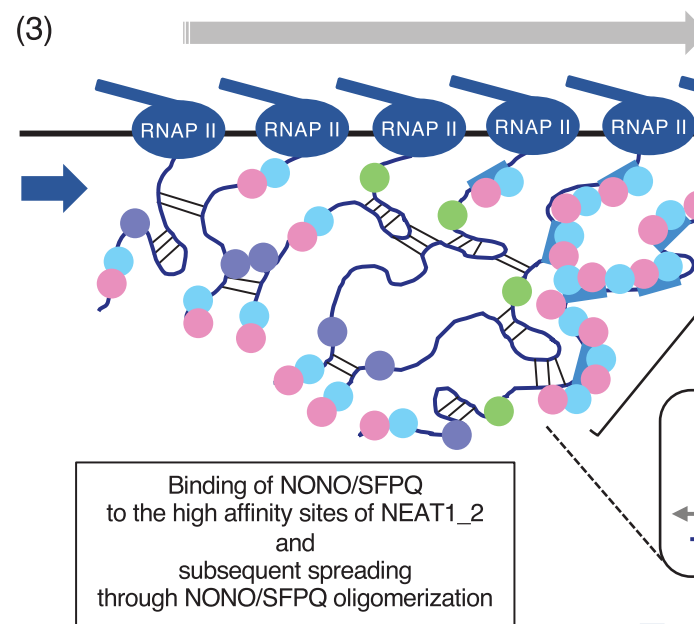

(4)

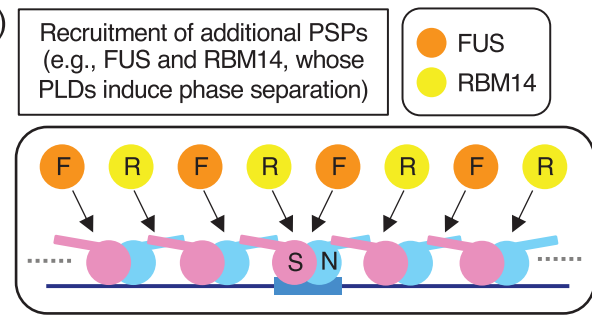

RNA-RNA interactions

Protein-protein interactions

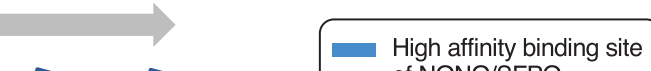
of NONO/SFPQ

(5) Formation of bundled/folded NEAT1_2 RNPs and

RNP assembly via multivalent interactions (e.g., PLDs), leading to phase separation
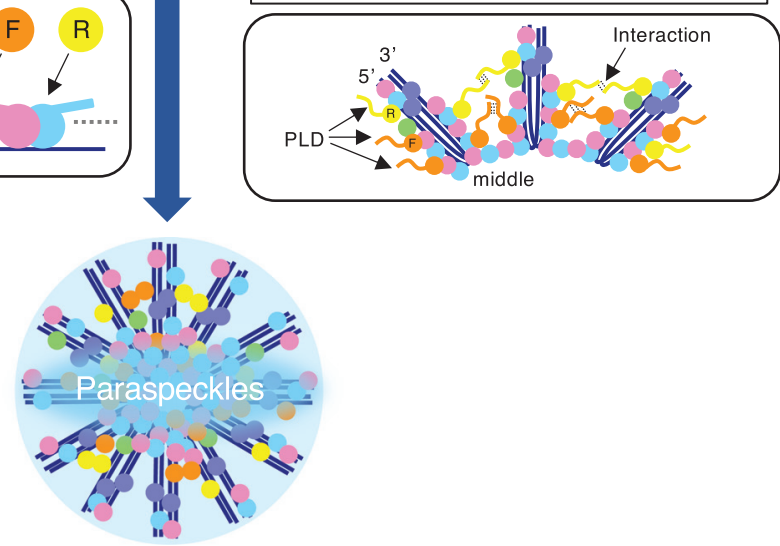

Figure 5. An updated model of paraspeckle formation showing the processes involved in NEAT1_2 transcription through paraspeckle formation via phase separation. See the main text for more details.

cellular differentiation (Nakagawa et al. 2014; Standaert et al. 2014; Wang et al. 2018; Modic et al. 2019). A number of studies have highlighted the importance of NEAT1 as a direct target of the p53 central tumor suppressor protein in cancer (Dong et al. 2018; Nakagawa et al. 2018). NEAT1 is also involved in various other pathological conditions, including neurodegenerative diseases and viral/bacterial infections (Nishimoto et al.
2013; Imamura et al. 2018). Additional research into the relationship between the RNA motifs of NEAT1 and its physiological functions is required to clarify the mechanisms of actions of arcRNAs and the biological significance of nuclear body formation on these molecules.

Several recent studies have shown that arcRNAs are still hidden within genomes. Using our recently developed 
method that relies on the semiextractable feature of arcRNAs, we performed a genome-wide screen of HeLa cells and identified 45 candidate arcRNAs (Chujo et al. 2017). These candidates include lncRNAs, pre-mRNAs, and repeat-derived RNAs and form nuclear foci that are distinct from known nuclear bodies (Chujo et al. 2017). In addition, another recent study showed that short tandem repeat-containing RNAs (strRNAs) might be a rich source of arcRNAs (Yap et al. 2018). One of the strRNAs is a core of the perinucleolar compartment. As most arcRNAs are induced by various stimuli and/or specific physiological or pathological conditions, searches performed under various conditions will likely expand the repertoire of known arcRNAs.

Because known arcRNAs have partner proteins, several approaches can be used to identify the components of arcRNA-induced cellular bodies. Most PSPs identified to date were found by colocalization screening using a cDNA library and fluorescent fusion proteins (Naganuma et al. 2012; Fong et al. 2013; Mannen et al. 2016). Our recent comprehensive ChIRP-MS (chromatin isolation by RNA purification followed by mass spectrometry) analysis revealed the compositions of nSBs, among which most of the proteins (141) were newly identified (Ninomiya et al. 2019). Proximity labeling methods such as APEX and BioID have also been used to determine the compositions and dynamic compositional changes of phase-separated cellular bodies (Markmiller et al. 2018; Youn et al. 2018; Padron et al. 2019). These approaches could be used to obtain a comprehensive list of the components and their dynamic exchanges within cellular bodies under various cellular conditions. Future studies should also reveal the mechanisms by which cellular body compositions are determined by arcRNAs and their motifs.

It is well known that arcRNAs can induce phase transitions between several material states (e.g., liquid, hydrogel, and solid) of cellular bodies. Exactly how these material properties are determined remains unanswered. In particular, it is unclear which protein components contribute to each state. The binding sites of the proteins on arcRNAs, including their affinities and densities, are still unknown, and the motifs and properties (e.g., amino acid compositions in PLDs, LCDs, or IDRs) of the proteins that determine the material states also require clarification. In vitro reconstitution of arcRNA-induced bodies using purified components or cellular/nuclear extracts should elucidate how they are constructed and how their biophysical properties are determined (Maharana et al. 2018). In addition to paraspeckles, other arcRNA-induced bodies also show various distinct morphologies. For example, nSBs display island structures that are composed of dense cores and shells (Kawaguchi et al. 2015), and amyloid bodies have filamentous structures (Audas et al. 2016). Therefore, it would be interesting to delineate how these morphologies are determined by arcRNAs and their partner proteins.

Identification of both the partner proteins and the precise binding sites as RNP modules should enable artificial control and reprogramming of arcRNAs. Motifspecific functional inhibition (or activation) might be achievable using antisense oligos, small molecules, or CRISPR-based strategies. In future analyses of arcRNAs, theoretical models and simulations, in addition to new methodologies and quantitative analyses, would offer novel frameworks to explain the mechanisms involved in complex biological phase separation.

\section{ACKNOWLEDGMENTS}

This work was supported by MEXT KAKENHI grants (to T.Y. [17K15058, 19K06479, 19H05250], S.N. [17H03604], and T.H. [JP26113002, JP16H06279, JP17H03630, and JP17K19335]), and by The Mochida Memorial Foundation for Medical and Pharmaceutical Research (to T.Y.), The Naito Foundation (to T.Y. and S.N.), The Akiyama Life Science Foundation (to T.Y.), Torey Research Fund (to S.N.), and Tokyo Biochemical Research Foundation (to T.H.).

\section{REFERENCES}

Adriaens C, Rambow F, Bervoets G, Silla T, Mito M, Chiba T, Hiroshi A, Hirose T, Nakagawa S, Jensen TH, et al. 2019. The lncRNA NEAT1_l is seemingly dispensable for normal tissue homeostasis and cancer cell growth. RNA 25: 1681-1695. doi:10.1261/rna.071456.119

Alberti S, Gladfelter A, Mittag T. 2019. Considerations and challenges in studying liquid-liquid phase separation and biomolecular condensates. Cell 176: 419-434. doi:10.1016/j.cell .2018 .12 .035

Aly MK, Ninomiya K, Adachi S, Natsume T, Hirose T. 2019. Two distinct nuclear stress bodies containing different sets of RNA-binding proteins are formed with HSATIII architectural noncoding RNAs upon thermal stress exposure. Biochem Biophys Res Commun 516: 419-423. doi:10.1016/j.bbrc.2019.06 .061

Audas TE, Audas DE, Jacob MD, Ho JJ, Khacho M, Wang M, Perera JK, Gardiner C, Bennett CA, Head T, et al. 2016. Adaptation to stressors by systemic protein amyloidogenesis. Dev Cell 39: 155-168. doi:10.1016/j.devcel.2016.09.002

Baltz AG, Munschauer M, Schwanhausser B, Vasile A, Murakawa Y, Schueler M, Youngs N, Penfold-Brown D, Drew K, Milek M, et al. 2012. The mRNA-bound proteome and its global occupancy profile on protein-coding transcripts. Mol Cell 46: 674-690. doi:10.1016/j.molcel.2012.05.021

Banani SF, Lee HO, Hyman AA, Rosen MK. 2017. Biomolecular condensates: organizers of cellular biochemistry. Nat Rev Mol Cell Biol 18: 285-298. doi:10.1038/nrm.2017.7

Bonetti A, Agostini F, Suzuki AM, Hashimoto K, Pascarella G, Gimenez J, Roos L, Nash AJ, Ghilotti M, Cameron CJF, et al. 2019. RADICL-seq identifies general and cell type-specific principles of genome-wide RNA-chromatin interactions. bioRxiv doi:10.1101/681924

Brown JA, Valenstein ML, Yario TA, Tycowski KT, Steitz JA. 2012. Formation of triple-helical structures by the 3 '-end sequences of MALAT1 and MEN $\beta$ noncoding RNAs. Proc Natl Acad Sci 109: 19202-19207. doi:10.1073/pnas.1217338109

Cai L, Chang H, Fang Y, Li G. 2016. A comprehensive characterization of the function of LincRNAs in transcriptional regulation through long-range chromatin interactions. Sci Rep 6: 36572. doi:10.1038/srep36572

Castello A, Fischer B, Eichelbaum K, Horos R, Beckmann BM, Strein C, Davey NE, Humphreys DT, Preiss T, Steinmetz LM, et al. 2012. Insights into RNA biology from an atlas of mammalian mRNA-binding proteins. Cell 149: 1393-1406. doi:10 $.1016 /$ j.cell.2012.04.031

Castello A, Fischer B, Frese CK, Horos R, Alleaume AM, Foehr S, Curk T, Krijgsveld J, Hentze MW. 2016. Comprehensive 
identification of RNA-binding domains in human cells. Mol Cell 63: 696-710. doi:10.1016/j.molcel.2016.06.029

Chen LL, Carmichael GG. 2009. Altered nuclear retention of mRNAs containing inverted repeats in human embryonic stem cells: functional role of a nuclear noncoding RNA. Mol Cell 35: 467-478. doi:10.1016/j.molcel.2009.06.027

Chu C, Zhang QC, da Rocha ST, Flynn RA, Bharadwaj M, Calabrese JM, Magnuson T, Heard E, Chang HY. 2015. Systematic discovery of Xist RNA binding proteins. Cell 161: 404-416. doi:10.1016/j.cell.2015.03.025

Chujo T, Hirose T. 2017. Nuclear bodies built on architectural long noncoding RNAs: unifying principles of their construction and function. Mol Cells 40: 889-896. doi:10.14348/mol cells.2017.0263

Chujo T, Yamazaki T, Hirose T. 2016. Architectural RNAs (arcRNAs): a class of long noncoding RNAs that function as the scaffold of nuclear bodies. Biochim Biophys Acta 1859: 139-146. doi:10.1016/j.bbagrm.2015.05.007

Chujo T, Yamazaki T, Kawaguchi T, Kurosaka S, Takumi T, Nakagawa S, Hirose T. 2017. Unusual semi-extractability as a hallmark of nuclear body-associated architectural noncoding RNAs. EMBO J 36: 1447-1462. doi:10.15252/embj .201695848

Clemson CM, Hutchinson JN, Sara SA, Ensminger AW, Fox AH, Chess A, Lawrence JB. 2009. An architectural role for a nuclear noncoding RNA: NEAT1 RNA is essential for the structure of paraspeckles. Mol Cell 33: 717-726. doi:10.1016/j .molcel.2009.01.026

Colognori D, Sunwoo H, Kriz AJ, Wang CY, Lee JT. 2019. Xist deletional analysis reveals an interdependency between Xist RNA and Polycomb complexes for spreading along the inactive X. Mol Cell 74: 101-117 e110. doi:10.1016/j.molcel.2019 .01 .015

Dong P, Xiong Y, Yue J, Hanley SJB, Kobayashi N, Todo Y, Watari H. 2018. Long non-coding RNA NEAT1: a novel target for diagnosis and therapy in human tumors. Front Genet 9: 471. doi:10.3389/fgene.2018.00471

Dumbović G, Biayna J, Banús J, Samuelsson J, Roth A, Diederichs S, Alonso S, Buschbeck M, Perucho M, Forcales SV. 2018. A novel long non-coding RNA from NBL2 pericentromeric macrosatellite forms a perinucleolar aggregate structure in colon cancer. Nucleic Acids Res 46: 55045-5524. doi:10 $.1093 /$ nar/gky263

Dumelie JG, Jaffrey SR. 2017. Defining the location of promoterassociated R-loops at near-nucleotide resolution using bisDRIP-seq. Elife 6: e28306. doi:10.7554/eLife.28306

Engreitz JM, Ollikainen N, Guttman M. 2016. Long non-coding RNAs: spatial amplifiers that control nuclear structure and gene expression. Nat Rev Mol Cell Biol 17: 756-770. doi:10 $.1038 / \mathrm{nrm} .2016 .126$

Fong KW, Li Y, Wang W, Ma W, Li K, Qi RZ, Liu D, Songyang Z, Chen J. 2013. Whole-genome screening identifies proteins localized to distinct nuclear bodies. J Cell Biol 203: 149164. doi:10.1083/jcb.201303145

Fox AH, Lam YW, Leung AK, Lyon CE, Andersen J, Mann M, Lamond AI. 2002. Paraspeckles: a novel nuclear domain. Curr Biol 12: 13-25. doi:10.1016/S0960-9822(01)00632-7

Fox AH, Bond CS, Lamond AI. 2005. P54nrb forms a heterodimer with PSP1 that localizes to paraspeckles in an RNAdependent manner. Mol Biol Cell 16: 5304-5315. doi:10 $.1091 / \mathrm{mbc} . \mathrm{e} 05-06-0587$

Fox AH, Nakagawa S, Hirose T, Bond CS. 2018. Paraspeckles: where long noncoding RNA meets phase separation. Trends Biochem Sci 43: 124-135. doi:10.1016/j.tibs.2017.12 .001

Geisler S, Coller J. 2013. RNA in unexpected places: long noncoding RNA functions in diverse cellular contexts. Nat Rev Mol Cell Biol 14: 699-712. doi:10.1038/nrm3679

Guttman M, Rinn JL. 2012. Modular regulatory principles of large non-coding RNAs. Nature 482: 339-346. doi:10.1038/ nature 10887

Hall LL, Byron M, Carone DM, Whitfield TW, Pouliot GP, Fischer A, Jones P, Lawrence JB. 2017. Demethylated HSATII
DNA and HSATII RNA foci sequester PRC1 and MeCP2 into cancer-specific nuclear bodies. Cell Rep 18: 2943-2956. doi:10.1016/j.celrep.2017.02.072

Hennig S, Kong G, Mannen T, Sadowska A, Kobelke S, Blythe A, Knott GJ, Iyer KS, Ho D, Newcombe EA, et al. 2015. Prion-like domains in RNA binding proteins are essential for building subnuclear paraspeckles. J Cell Biol 210: 529-539. doi: $10.1083 /$ jcb. 201504117

Hirose T, Mishima Y, Tomari Y. 2014a. Elements and machinery of non-coding RNAs: toward their taxonomy. EMBO Rep 15: 489-507. doi:10.1002/embr.201338390

Hirose T, Virnicchi G, Tanigawa A, Naganuma T, Li R, Kimura H, Yokoi T, Nakagawa S, Bénard M, Fox AH, et al. 2014b. NEAT1 long noncoding RNA regulates transcription via protein sequestration within subnuclear bodies. Mol Biol Cell 25: 169-183. doi:10.1091/mbc.e13-09-0558

Hirose T, Yamazaki T, Nakagawa S. 2019. Molecular anatomy of the architectural NEAT1 noncoding RNA: the domains, interactors, and biogenesis pathway required to build phase-separated nuclear paraspeckles. Wiley Interdiscip Rev RNA 10: e1545. doi:10.1002/wrna.1545

Huang J, Casas Garcia GP, Perugini MA, Fox AH, Bond CS, Lee M. 2018. Crystal structure of a SFPQ/PSPC1 heterodimer provides insights into preferential heterodimerization of human DBHS family proteins. J Biol Chem 293: 6593-6602. doi:10 $.1074 /$ jbc.RA117.001451

Imamura K, Imamachi N, Akizuki G, Kumakura M, Kawaguchi A, Nagata K, Kato A, Kawaguchi Y, Sato H, Yoneda M, et al. 2014. Long noncoding RNA NEAT1-dependent SFPQ relocation from promoter region to paraspeckle mediates IL8 expression upon immune stimuli. Mol Cell 53: 393-406. doi:10 $.1016 /$ j.molcel.2014.01.009

Imamura K, Takaya A, Ishida YI, Fukuoka Y, Taya T, Nakaki R, Kakeda M, Imamachi N, Sato A, Yamada T, et al. 2018. Diminished nuclear RNA decay upon Salmonella infection upregulates antibacterial noncoding RNAs. EMBO J 37: e97723. doi:10.15252/embj.201797723

Isobe M, Toya H, Mito M, Chiba T, Asahara H, Hirose T, Nakagawa S. 2019. Forced isoform switching of Neat1 1 to Neat1_2 leads to the loss of Neat1_1 and the hyperformation of paraspeckles but does not affect the development and growth of mice. $R N A$ doi:10.1261/rna.072587.119

Jiang L, Shao C, Wu QJ, Chen G, Zhou J, Yang B, Li H, Gou LT, Zhang Y, Wang Y, et al. 2017. NEAT1 scaffolds RNA-binding proteins and the Microprocessor to globally enhance primiRNA processing. Nat Struct Mol Biol 24: 816-824. doi: $10.1038 / \mathrm{nsmb} .3455$

Jolly C, Usson Y, Morimoto RI. 1999. Rapid and reversible relocalization of heat shock factor 1 within seconds to nuclear stress granules. Proc Natl Acad Sci 96: 6769-6774. doi:10 $.1073 /$ pnas.96.12.6769

Kawaguchi T, Tanigawa A, Naganuma T, Ohkawa Y, Souquere S, Pierron G, Hirose T. 2015. SWI/SNF chromatin-remodeling complexes function in noncoding RNA-dependent assembly of nuclear bodies. Proc Natl Acad Sci 112: 4304-4309. doi:10 $.1073 /$ pnas. 1423819112

Lee M, Sadowska A, Bekere I, Ho D, Gully BS, Lu Y, Iyer KS, Trewhella J, Fox AH, Bond CS. 2015. The structure of human SFPQ reveals a coiled-coil mediated polymer essential for functional aggregation in gene regulation. Nucleic Acids Res 43: 3826-3840. doi:10.1093/nar/gkv156

Li R, Harvey AR, Hodgetts SI, Fox AH. 2017a. Functional dissection of NEAT1 using genome editing reveals substantial localization of the NEAT1_1 isoform outside paraspeckles. RNA 23: 872-881. doi:10.1261/rna.059477.116

Li X, Zhou B, Chen L, Gou LT, Li H, Fu XD. 2017b. GRID-seq reveals the global RNA-chromatin interactome. Nat Biotechnol 35: 940-950. doi:10.1038/nbt.3968

Lin Y, Schmidt BF, Bruchez MP, McManus CJ. 2018. Structural analyses of NEAT1 lncRNAs suggest long-range RNA interactions that may contribute to paraspeckle architecture. Nucleic Acids Res 46: 3742-3752. doi:10.1093/nar/ gky046 
Lu Z, Zhang QC, Lee B, Flynn RA, Smith MA, Robinson JT, Davidovich C, Gooding AR, Goodrich KJ, Mattick JS, et al. 2016. RNA duplex map in living cells reveals higher-order transcriptome structure. Cell 165: 1267-1279. doi:10.1016/j .cell.2016.04.028

Maharana S, Wang J, Papadopoulos DK, Richter D, Pozniakovsky A, Poser I, Bickle M, Rizk S, Guillen-Boixet J, Franzmann TM, et al. 2018. RNA buffers the phase separation behavior of prion-like RNA binding proteins. Science 360: 918-921. doi:10.1126/science.aar7366

Mallik M, Lakhotia SC. 2009. RNAi for the large non-coding hsrw transcripts suppresses polyglutamine pathogenesis in Drosophila models. RNA Biol 6: 464-478. doi:10.4161/rna .6.4.9268

Mannen T, Yamashita S, Tomita K, Goshima N, Hirose T. 2016. The Sam68 nuclear body is composed of two RNase-sensitive substructures joined by the adaptor HNRNPL. J Cell Biol 214: 45-59. doi:10.1083/jcb.201601024

Mao YS, Sunwoo H, Zhang B, Spector DL. 2011. Direct visualization of the co-transcriptional assembly of a nuclear body by noncoding RNAs. Nat Cell Biol 13: 95-101. doi:10.1038/ ncb2140

Markmiller S, Soltanieh S, Server KL, Mak R, Jin W, Fang MY, Luo EC, Krach F, Yang D, Sen A, et al. 2018. Context-dependent and disease-specific diversity in protein interactions within stress granules. Cell 172: 590-604 e513. doi:10.1016/j.cell .2017 .12 .032

Modic M, Grosch M, Rot G, Schirge S, Lepko T, Yamazaki T, Lee FCY, Rusha E, Shaposhnikov D, Palo M, et al. 2019. Cross-regulation between TDP-43 and paraspeckles promotes pluripotency-differentiation transition. Mol Cell 74: 951-965. doi:10.1016/j.molcel.2019.03.041

Monfort A, Wutz A. 2017. Progress in understanding the molecular mechanism of Xist RNA function through genetics. Philos Trans R Soc Lond B Biol Sci 372: 20160368. doi:10 $.1098 /$ rstb. 2016.0368

Naganuma T, Nakagawa S, Tanigawa A, Sasaki YF, Goshima N, Hirose T. 2012. Alternative 3'-end processing of long noncoding RNA initiates construction of nuclear paraspeckles. $E M B O$ J 31: 4020-4034. doi:10.1038/emboj.2012.251

Nakagawa S, Shimada M, Yanaka K, Mito M, Arai T, Takahashi E, Fujita Y, Fujimori T, Standaert L, Marine JC, et al. 2014. The lncRNA Neatl is required for corpus luteum formation and the establishment of pregnancy in a subpopulation of mice. Development 141: 4618-4627. doi:10.1242/dev .110544

Nakagawa S, Yamazaki T, Hirose T. 2018. Molecular dissection of nuclear paraspeckles: towards understanding the emerging world of the RNP milieu. Open Biol 8: 180150. doi:10.1098/ rsob. 180150

Ninomiya K, Adachi S, Natsume T, Iwakiri J, Terai G, Asai K, Hirose T. 2019. LncRNA-dependent nuclear stress bodies promote intron retention through SR protein phosphorylation. EMBO J e102729. doi:10.15252/embj.2019102729

Nishimoto Y, Nakagawa S, Hirose T, Okano HJ, Takao M, Shibata S, Suyama S, Kuwako K, Imai T, Murayama S, et al. 2013. The long non-coding RNA nuclear-enriched abundant transcript 1_2 induces paraspeckle formation in the motor neuron during the early phase of amyotrophic lateral sclerosis. Mol Brain 6: 31. doi:10.1186/1756-6606-6-31

Padron A, Iwasaki S, Ingolia NT. 2019. Proximity RNA labeling by APEX-seq reveals the organization of translation initiation complexes and repressive RNA granules. Mol Cell 75: 875-887.e875. doi:10.1016/j.molcel.2019.07.030

Passon DM, Lee M, Rackham O, Stanley WA, Sadowska A, Filipovska A, Fox AH, Bond CS. 2012. Structure of the heterodimer of human NONO and paraspeckle protein component 1 and analysis of its role in subnuclear body formation. Proc Natl Acad Sci 109: 4846-4850. doi:10.1073/pnas .1120792109

Peng A, Weber SC. 2019. Evidence for and against liquid-liquid phase separation in the nucleus. Noncoding RNA 5: E50. doi: $10.3390 /$ ncrna5040050
Quinn JJ, Chang HY. 2016. Unique features of long non-coding RNA biogenesis and function. Nat Rev Genet 17: 47-62. doi:10.1038/nrg.2015.10

Sasaki YT, Ideue T, Sano M, Mituyama T, Hirose T. 2009. MEN $\varepsilon / \beta$ noncoding RNAs are essential for structural integrity of nuclear paraspeckles. Proc Natl Acad Sci 106: 2525-2530. doi:10.1073/pnas.0807899106

Schmitt AM, Chang HY. 2017. Long noncoding RNAs: at the intersection of cancer and chromatin biology. Cold Spring Harb Perspect Med 7: a026492. doi:10.1101/cshperspect .a026492

Senturk Cetin N, Kuo CC, Ribarska T, Li R, Costa IG, Grummt I. 2019. Isolation and genome-wide characterization of cellular DNA:RNA triplex structures. Nucleic Acids Res 47: 23062321. doi:10.1093/nar/gky1305

Shevtsov SP, Dundr M. 2011. Nucleation of nuclear bodies by RNA. Nat Cell Biol 13: 167-173. doi:10.1038/ncb2157

Shin Y, Brangwynne CP. 2017. Liquid phase condensation in cell physiology and disease. Science 357: 1253. doi:10.1126/sci ence.aaf4382

Souquere S, Beauclair G, Harper F, Fox A, Pierron G. 2010. Highly ordered spatial organization of the structural long noncoding NEAT1 RNAs within paraspeckle nuclear bodies. $\mathrm{Mol}$ Biol Cell 21: 4020-4027. doi:10.1091/mbc.e10-08-0690

Standaert L, Adriaens C, Radaelli E, Van Keymeulen A, Blanpain C, Hirose T, Nakagawa S, Marine JC. 2014. The long noncoding RNA Neat 1 is required for mammary gland development and lactation. RNA 20: 1844-1849. doi:10.1261/rna .047332 .114

Sunwoo H, Dinger ME, Wilusz JE, Amaral PP, Mattick JS, Spector DL. 2009. $M E N \varepsilon / \beta$ nuclear-retained non-coding RNAs are up-regulated upon muscle differentiation and are essential components of paraspeckles. Genome Res 19: 347-359. doi:10.1101/gr.087775.108

Tollervey JR, Curk T, Rogelj B, Briese M, Cereda M, Kayikci M, Konig J, Hortobagyi T, Nishimura AL, Zupunski V, et al. 2011. Characterizing the RNA targets and position-dependent splicing regulation by TDP-43. Nat Neurosci 14: 452-458. doi:10 $.1038 / \mathrm{nn} .2778$

Van Nostrand EL, Pratt GA, Shishkin AA, Gelboin-Burkhart C, Fang MY, Sundararaman B, Blue SM, Nguyen TB, Surka C, Elkins K, et al. 2016. Robust transcriptome-wide discovery of RNA-binding protein binding sites with enhanced CLIP (eCLIP). Nat Methods 13: 508-514. doi:10.1038/nmeth.3810

Van Treeck B, Parker R. 2018. Emerging roles for intermolecular RNA-RNA interactions in RNP assemblies. Cell 174: 791802. doi:10.1016/j.cell.2018.07.023

Visa N, Puvion-Dutilleul F, Bachellerie JP, Puvion E. 1993. Intranuclear distribution of U1 and U2 snRNAs visualized by high resolution in situ hybridization: revelation of a novel compartment containing U1 but not U2 snRNA in HeLa cells. Eur $J$ Cell Biol 60: 308-321.

Wang Y, Hu SB, Wang MR, Yao RW, Wu D, Yang L, Chen LL. 2018. Genome-wide screening of NEAT1 regulators reveals cross-regulation between paraspeckles and mitochondria. Nat Cell Biol 20: 1145-1158. doi:10.1038/s41556018-0204-2

West JA, Davis CP, Sunwoo H, Simon MD, Sadreyev RI, Wang PI, Tolstorukov MY, Kingston RE. 2014. The long noncoding RNAs NEAT1 and MALAT1 bind active chromatin sites. Mol Cell 55: 791-802. doi:10.1016/j.molcel.2014.07.012

West JA, Mito M, Kurosaka S, Takumi T, Tanegashima C, Chujo T, Yanaka K, Kingston RE, Hirose T, Bond C, et al. 2016. Structural, super-resolution microscopy analysis of paraspeckle nuclear body organization. J Cell Biol 214: 817830. doi:10.1083/jcb.201601071

Wilusz JE, JnBaptiste CK, Lu LY, Kuhn CD, Joshua-Tor L, Sharp PA. 2012. A triple helix stabilizes the $3^{\prime}$ ends of long noncoding RNAs that lack poly(A) tails. Genes Dev 26: 2392-2407. doi:10.1101/gad.204438.112

Wutz A, Rasmussen TP, Jaenisch R. 2002. Chromosomal silencing and localization are mediated by different domains of Xist RNA. Nat Genet 30: 167-174. doi:10.1038/ng820 
Yamashita A, Watanabe Y, Nukina N, Yamamoto M. 1998. RNAassisted nuclear transport of the meiotic regulator Mei2p in fission yeast. Cell 95: 115-123. doi:10.1016/S0092-8674 (00)81787-0

Yamazaki T, Hirose T. 2015. The building process of the functional paraspeckle with long non-coding RNAs. Front Biosci (Elite Ed) 7: 1-41. doi:10.2741/s420

Yamazaki T, Fujikawa C, Kubota A, Takahashi A, Hirose T 2018a. CRISPRa-mediated NEAT1 lncRNA upregulation induces formation of intact paraspeckles. Biochem Biophys Res Commun 504: 218-224. doi:10.1016/j.bbrc.2018.08.158

Yamazaki T, Souquere S, Chujo T, Kobelke S, Chong YS, Fox AH, Bond CS, Nakagawa S, Pierron G, Hirose T. 2018b. Functional domains of NEAT1 architectural lncRNA induce paraspeckle assembly through phase separation. $\mathrm{Mol}$ Cell 70: 1038-1053.e1037. doi:10.1016/j.molcel.2018.05 .019

Yang L-Z, Wang Y, Li S-Q, Yao R-W, Luan P-F, Wu H, Carmichael GG, Chen L-L. 2019. Dynamic imaging of RNA in living cells by CRISPR-Cas13 systems. Mol Cell doi:10 .1016/j.molcel.2019.10.024

Yap K, Mukhina S, Zhang G, Tan JSC, Ong HS, Makeyev EV. 2018. A short tandem repeat-enriched RNA assembles a nuclear compartment to control alternative splicing and promote cell survival. Mol Cell 72: 525-540.e513. doi:10.1016/j .molcel.2018.08.041

Youn JY, Dunham WH, Hong SJ, Knight JDR, Bashkurov M, Chen GI, Bagci H, Rathod B, MacLeod G, Eng SWM, et al. 2018. High-density proximity mapping reveals the subcellular organization of mRNA-associated granules and bodies. Mol Cell 69: 517-532.e511. doi:10.1016/j.molcel.2017.12.020

Zhang N, Ashizawa T. 2017. RNA toxicity and foci formation in microsatellite expansion diseases. Curr Opin Genet Dev 44: 17-29. doi:10.1016/j.gde.2017.01.005

Zhu J, Mayeda A, Krainer AR. 2001. Exon identity established through differential antagonism between exonic splicing silencer-bound hnRNP A1 and enhancer-bound SR proteins. Mol Cell 8: 1351-1361. doi:10.1016/S1097-2765(01)00409-9 


\section{$\$_{\text {CSH }}^{\infty}$ Cold Spring Harbor Symposia SYMPOSIA on Quantitative Biology}

\section{Architectural RNAs for Membraneless Nuclear Body Formation}

Tomohiro Yamazaki, Shinichi Nakagawa and Tetsuro Hirose

Cold Spring Harb Symp Quant Biol published online February 4, 2020

Access the most recent version at doi:10.1101/sqb.2019.84.039404

$\mathbf{P}<\mathbf{P} \quad$ Published online February 4, 2020 in advance of the print journal.

Creative This article is distributed under the terms of the

Commons http://creativecommons.org/licenses/by-nc/4.0/, which permits reuse and

License redistribution, except for commercial purposes, provided that the original author and source are credited.

Email Alerting Receive free email alerts when new articles cite this article - sign up in Service the box at the top right corner of the article or click here.

Advance online articles have been peer reviewed and accepted for publication but have not yet appeared in the paper journal (edited, typeset versions may be posted when available prior to final publication). Advance online articles are citable and establish publication priority; they are indexed by PubMed from initial publication. Citations to Advance online articles must include the digital object identifier (DOIs) and date of initial publication.

To subscribe to Cold Spring Harbor Symposia on Quantitative Biology go to: http://symposium.cshlp.org/subscriptions 